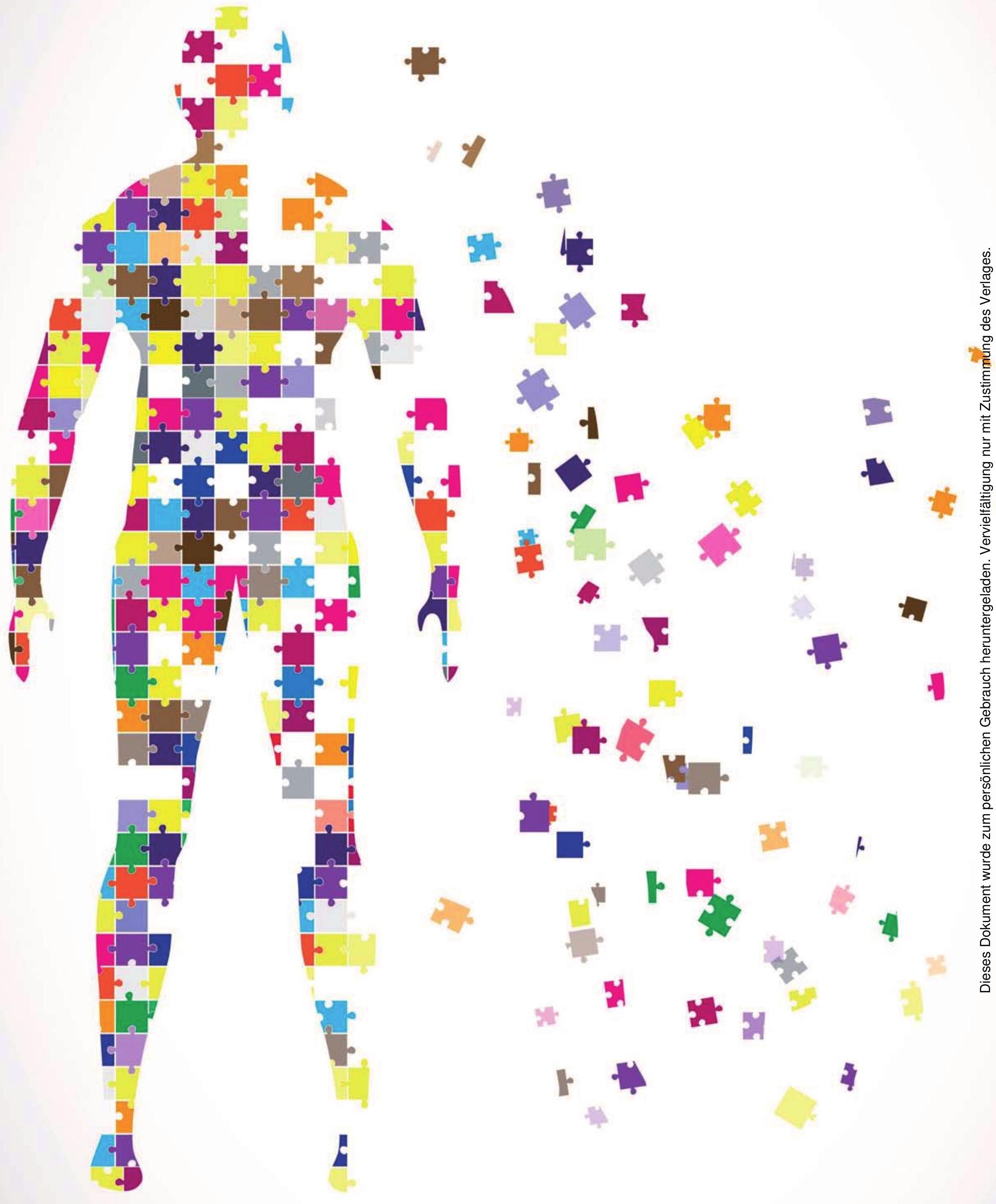




\title{
Von Gedankenblüten und Erkenntnisblitzen
}

\author{
GANZHEITLICHE DIAGNOSTIK in der Naturheilpraxis: \\ Den Menschen wahrnehmen - über die Laborwerte hinaus \\ Christina Casagrande
}

ICH WAR 17 JAHRE jung und meine Mutter bestand auf eine Diphtherie-Impfung. Sie selbst wäre als Kind fast an dieser Krankheit gestorben. Meine Schwester und ich wurden mit dem Impfserum zu unserem Hausarzt geschickt.

\section{Der alte Hausarzt}

Ich kann mich gut erinnern: Er holte seine Patienten immer persönlich aus dem Wartezimmer. Dieses Mal blieb er kurz auf der Türschwelle stehen, fixierte mich mit seinen wasserblauen Augen und bat meine Schwester zu warten. Er gab mir die Hand und fragte unvermittelt: „Hast Du Liebeskummer?“ Ich bekam einen roten Kopf und wies das weit von mir. „Wirklich nicht?“ - „Nein, ich habe keinen Freund zurzeit.“ - „Hast Du geweint?“ - „Nein!“ Was sollte die ganze Fragerei? Ich war hier, um geimpft zu werden, und nicht, um kleine Sünden zu beichten! „Geh auf die Toilette und lass eine Urinprobe da. Du wirst nicht geimpft. Du gehst jetzt nach Hause und legst Dich ins Bett. Alles Weitere bespreche ich mit Deiner Mutter“, sagte er daraufhin. Verstört ging ich nach Hause. Meine Mutter erhielt von ihm die Anweisung, mir salzarme Kost zu geben und darauf zu achten, dass ich viel trank. In einer Woche sollte ich mich wieder in der Praxis vorstellen. Drei Tage später trat die erste Nierenkolik auf, eine Röntgenuntersuchung ergab, dass ich einen Nierenstein hatte.

Was hatte der Hausarzt wahrgenommen? War es die Intuition eines lang praktizierenden Arztes? War er hellsichtig?

\section{Sein Erfolgsgeheimnis}

Zwei Jahre später begann ich bei ihm als Arzthelferin zu arbeiten. Ich nahm allen Mut zusammen und fragte ihn, was ihn damals dazu veranlasst hatte, mich ins Bett zu stecken. Er schmunzelte: „Du hast nach Niere gerochen, Deine Augen waren geschwollen, Deine Hände kühl-klamm und Du hattest dunkle Schatten unter den Augen. Da wusste ich, Bettruhe schadet keinesfalls. Erythrozyten und Eiweiß im Urin bestätigten meine Vermutung, dass etwas mit Deinen Nieren nicht in Ordnung war. An einen Nierenstein, wie sich dann auf dem Röntgenbild zeigte, dachte ich zu diesem Zeitpunkt noch nicht.“

Ich habe unseren alten Hausarzt nie vergessen und bewundere ihn heute noch. Er praktizierte unspektakulär, das Labor erinnerte an Mittelalter, die weiteren medizinischen Einrichtungen waren auf minimalem Standard. Und dennoch: Er half vielen

\section{KURZ GEFASST}

1 Das Ziel der ganzheitlichen Diagnostik ist es, den Patienten über konventionelle, apparative und Labordiagnostik hinaus in seiner Ganzheit zu erfassen.

7 Die Intuition ist dabei das Hauptwerkzeug des Heilpraktikers.

$\angle$ Der Verstand unterstützt diesen Prozess.

3 Anregungen und praktische Tipps sollen Ihnen helfen, die Mög3 lichkeiten ganzheitlicher Diagnostik in Ihrer Arbeit kennenzulernen und zu vertiefen.

Menschen, erkannte ihre Not oft schon, bevor sie diese selbst bemerkten. Zwar war er daher vielen Patienten mit seinen Anweisungen unbequem, doch seine Heilerfolge sprachen für ihn.

Genau so wollte ich auch Menschen helfen können. Damals hatte ich am eigenen Leib erfahren, was ganzheitliche Diagnostik ist, was sie kann und wie sie - im besten Fall - Hand in Hand mit konventioneller Diagnostik geht.

\section{Den Menschen ganzheitlich erfassen - aber wie?}

Noch nie standen Ärzten und Heilpraktikern so detaillierte und präzise Diagnosemöglichkeiten zur Verfügung wie heute. Labor und bildgebende Verfahren vermitteln uns den Eindruck, in den Menschen hineinblicken, jede Regung des Organismus verfolgen zu können bis hinunter auf die Ebene der Moleküle. Ja, es ist faszinierend. Ich selbst nutze die Ergebnisse dieser Diagnosemöglichkeiten, alles andere wäre fahrlässig.

Doch es gibt Situationen, in denen uns diese Bilder und Zahlen eine trügerische Sicherheit vermitteln. Immer dann, wenn wir vergessen, dass sie Momentaufnahmen dynamischer Prozesse sind und nicht ein unveränderlicher Sachverhalt. Wenn wir außer Acht lassen, dass weit im Vorfeld einer manifesten Erkrankung Zeichen auftauchen, die über Laborwerte oder bildgebende Verfahren nicht zu erfassen sind. Wenn wir vergessen, dass diese Untersuchungsmethoden Befunde zeigen, nicht aber die endgültige Diagnose und schon gar nicht die Therapie darstellen. 


\section{„Die Intuition ist ein göttliches Geschenk, der denkende Verstand ein treuer Diener. Es ist paradox, dass wir heute angefangen haben, den Diener zu verehren und die göttliche Gabe zu entweihen. “}

\section{Albert Einstein}

Sie finden hier Anregungen, um den ganzen Menschen in seinem Leid zu erfassen, jenseits und zusätzlich zu Labor- und apparativer Diagnostik oder bildgebenden Verfahren. Die Sternstunde der ganzheitlichen Diagnostik schlägt dann, wenn Sie die Gefahr einer Erkrankung erkennen, bevor sie sich auf der Körperebene manifestiert und/oder in Laborbefunden bestätigt. In der TCM gilt als der talentierteste Heiler jener, der seinen Patienten gesund erhält. Unspektakulär, nicht wahr?

\section{Zutat Zeit}

Nehmen Sie sich ausreichend Zeit für die Diagnose? Zeit ist eine wichtige Zutat. Sie wird meist zu sparsam, manchmal zu verschwenderisch eingesetzt. Der Patient soll weder aus Zeitnot etwas Wichtiges übergehen, noch soll er sich mit dem Therapeuten in Nebensächlichkeiten verirren. Ein sinnvoller Umgang mit Zeit bedeutet: ausreichend Zeit für das Patientengespräch, für Beratung am Telefon, für Zwischentöne. Auch Zeit für tröstende Worte, die oft Verborgenes hervorholen, Zeit, um den ganzen Patienten wahrzunehmen.

Neben den einfachen, auch in der Heilpraxis üblichen Diagnosemöglichkeiten wie Blutdruck und Puls messen, nervale Reflexe testen, Mundraum inspizieren, gilt es auch z. B.
- die Sprache der Haut wahrzunehmen,

- den spezifischen Körpergeruch des Patienten zu registrieren,

- Reflexzonen am Körper zu inspizieren oder zu ertasten,

- die Inspektion von z. B. Augen, Zunge, Fingernägeln mit in die Beurteilung des Gesamtbefindens etc. einzubeziehen.

Diese Liste der Grunddiagnostik lässt sich lange fortführen. Die Frage ist: Tun wir dies heute noch in unseren Praxen? Oder verlassen wir uns nur noch auf die Aussagen der Geräte- und Labordiagnostik, die viele Patienten zum Erstgespräch mitbringen? Wagen wir es, diese Ergebnisse zu hinterfragen oder ersetzen sie tatsächlich unsere eigene Wahrnehmung?

\section{Wahrnehmung der anderen Art: Intuition}

Unter Intuition verstehen wir Gedanken oder Einfälle, die wir rational nicht erklären können. Wir wissen nicht, woher sie kommen. Sie steigen wie Gedankenblüten in uns auf oder durchfahren uns wie Erkenntnisblitze. Die Dinge fallen an ihren Platz, Bilder oder Worte fügen sich stimmig. Intuition geschieht, wenn wir uns selbst vergessen, wenn $\mathrm{Zu}$ - und Abneigungen keine Rolle spielen. Wenn wir emotional sind, ist uns der intuitive Zugang zur Situation verwehrt.

\section{Beispiele intuitiver Diagnostik}

Eine erfahrene Osteopathin bezog sich bei der Antwort auf meine Frage, wie sie diagnostiziere, auf Rollin Becker, den Begründer und Leiter des Cranial Osteopathy Institutes in Großbritannien: „Osteopathen unterscheiden zwischen: Was der Patient meint, was mit ihm los ist, und was wir meinen, was mit ihm los ist. Worauf es jedoch ankommt und wonach wir uns idealerweise in der Diagnostik und Therapie richten: Wir hören seinem Organismus mit unserem eigenen gesamten System zu, hören, was der Organismus sagt - denn er ist intelligenter als wir und weiß es am besten. “ Diese Vorgehensweise habe ich selbst als Patientin erfahren dürfen - und ich war überrascht, was mein Körper der Osteopathin alles anvertraute.

Eine Atemtherapeutin, mit vielen Jahren Berufserfahrung, formulierte ihre ganzheitliche Diagnostik als ein Sehen des Atems anhand der feinen Bewegungen des Brustkorbs, ein Hören des Atems am Stimmklang und ein Erspüren des Patientenatems in sich selbst. Dazu lässt sie den Patienten malen und sich dabei auf Einund Ausatmen konzentrieren. In sich selbst verfolgt sie die Malbewegungen, atmet mit dem Patienten und erspürt krankmachende Blockaden auf diese Weise mit faszinierender Treffsicherheit.

Wenn wir bei der Diagnostik das Gottesgeschenk Intuition unseren Patienten zugute kommen lassen wollen, stellt sich folgende Frage. 


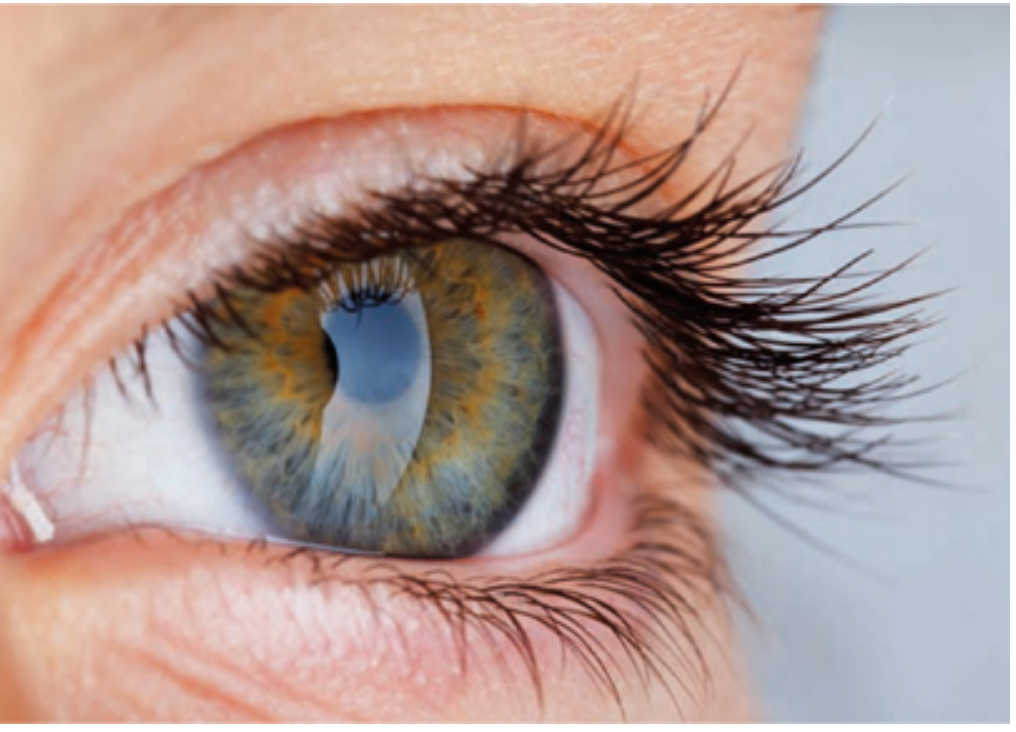

Foto: () Fotolia/Taffi

\section{Wie finde und entwickle ich Intuition?}

Bei der Begegnung mit Menschen müssen wir lernen, der ersten Wahrnehmung, die in uns aufsteigt, zu vertrauen. Dieses Vertrauen wird stark, wenn sich diese erste Wahrnehmung bei späteren Begegnungen faktisch bestätigt. Damit das zuverlässig gelingt, wir also intuitiv das Wesentliche wahrnehmen, müssen wir uns immer wieder selbst klären, mental und emotional.

Bei intuitiver Vorgehensweise ist es wichtig, in sich zu ruhen. Anziehung oder Ablehnung sind Emotionen, die unsere Wahrnehmung verfälschen. Reagieren wir beim Erstgespräch auf einen Patienten emotional, können wir uns bei der Diagnostik nicht auf unsere Intuition verlassen. In solchen Situationen hilft uns Albert Einsteins „treuer Diener“: unser Verstand - und systematisches Vorgehen. Idealerweise haben wir jedoch an uns gearbeitet und zu einer starken inneren Mitte gefunden, einem Ort, in dem wir emotionslos ruhen können.

\section{Die innere Mitte des Therapeuten}

Eine starke innere Mitte ist vorhanden, wenn es gelingt, in emotionalen Situationen die Lage vorurteilsfrei zu betrachten. Wenn Ängste, Ab- und Zuneigungen, Begeisterung und Befürchtungen im Entstehen erkannt werden und Gefühle uns nicht davontragen, handeln wir aus unserer inneren Mitte heraus.

Neben Entspannungs- und Konzentrationsübungen ist Authentizität wichtig. Weshalb? Wenn ich konsequent nur das empfehle, was ich selbst zu tun bereit bin, entwickle ich eine verlässliche innere Messlatte. Sie ist der persönliche Nullmeridian, auf den ich mich immer zurückbesinnen kann. Dieser Ankerpunkt, gepaart mit der Fähigkeit, Ruhe auch in Krisensituationen zu bewahren, sowie Ordnung in der eigenen Gedankenwelt machen eine starke Mitte aus. Ohne die ist eine zuverlässige Intuition nicht möglich.

\section{Das Werkzeug der Intuition}

Wenn Albert Einstein die Intuition als göttliches Geschenk bezeichnet, etwas Geistiges also, dann muss der Therapeut der Resonanzboden dafür sein. Um in Resonanz zu gehen, muss ich zunächst wahrnehmen können. Dies geschieht über die Sinnesorgane. Danach erfolgt die Umsetzung des Wahrgenommenen über das Wesen und Können des Therapeuten. Hierzu benötigt man klar funktionierende Sinne. Seh- und Tastsinn bedürfen täglicher Pflege, und insbesondere der Geruchsinn muss geschult werden, denn er verbindet uns mit dem limbischen System, unserer emotionalen Heimat im Kopf.

Wenn ich als Therapeut einen dieser genannten Sinne nicht voll funktionsfähig zur Verfügung habe, bedeutet dies nicht, dass ich nicht gut und intuitiv diagnostizieren kann. Ich muss mir der Sinneseinschränkung nur bewusst sein und alle anderen mir zur Verfügung stehenden Sinne so optimal wie möglich nutzen.

All unsere Sinne sind dankbar für ausreichend Schlaf und rhythmische Lebensführung. Idealerweise wechseln sich Aktivitäten und Erholungsphasen in kurzen Zeitabständen ab: den Tagesablauf 2- bis 3-mal mit einer 20-minütigen Pause zu unterbrechen bringt deutlich mehr, als einen Tag am Wochenende zu verschlafen. Weitere praktische Tipps für die Hygiene und Stärkung ihrer Sinne:

- Vermeiden Sie künstliche Duftstoffe, z. B. in Kosmetika vorhanden.

- Verwenden Sie keine künstlichen Geschmacksverstärker beim Kochen und verzichten Sie auf Fertiggerichte.

- Stark reizende Genussmittel, z. B. Kaffee, Alkohol, Süßigkeiten, sollten Sie, wenn überhaupt, nur sparsam genießen.

- Schauen Sie jeden Tag einmal bewusst ins Grüne. Bauen Sie Augenübungen aus Yoga, Feldenkrais oder anderen bewährten Methoden in den Alltag ein.

- Führen Sie wenigstens an einem Tag der Woche Medienfasten durch. Statt Lesen am Abend auch mal Hörbuch hören.

- Lauschen Sie der Natur: Bienensummen, Blätterrascheln, das Singen des Windes. Hören Sie leise klassische Musik. Achten Sie im Alltag auf die Unterschiedlichkeit von Stimmen und Atem.

- Nehmen Sie mit geschlossenen Augen die haptische Verschiedenartigkeit von Bekleidung wahr. Empfangen und geben Sie Streicheleinheiten. Gehen Sie bewusst barfuß auf verschiedenem Untergrund. Lehnen Sie sich an verschiedenen Orten zurück und nehmen Sie dabei die Verschiedenartigkeit von Bäumen, Felsen etc. wahr.

\section{Der treue Diener der Intuition: Der Verstand}

Neben der Wahrnehmung des Patienten mit allen Sinnen ist eine rationale und methodische Vorgehensweise hilfreich, mit welcher der emotionale Zustand des Patienten schnell erfasst wird. In meiner Praxis erfrage ich z. B. beim Erstgespräch die Lebensumstände des Patienten sowie seine Erwartungen und Wünsche an mich:

- Gibt es Zufriedenheit im Privat- und Berufsleben?

- Welche Lebensform wird gepflegt: alleine, in Partnerschaft, in einer Gemeinschaft etc.?

- Wie ist das augenblickliche Lebensgefühl?

- Gibt es Ziele im Leben, und wenn ja, welche?

- Was ist das Ziel unserer Zusammenarbeit?

- Was ist der Auftrag des Patienten an mich?

\section{Analogien sind Wegweiser}

Mit geschärften Sinnen und klärenden Fragen erhalten Sie deutlich mehr Informationen. Diese gilt es rasch zu ordnen, ohne dabei den Patienten in eine Schablone zu pressen. Dabei hilft das 


\section{Für den Praxisalltag}

Haben Sie das Gefühl, dass Sie sich von den Problemen Ihrer Patienten schlecht abgrenzen können? Da es für jedes Gift ein Gegengift gibt, will ich mit Ihnen 2 meiner erprobten Hilfsmittel teilen:

Schutz-Öl: In 10 ml Jojobaöl geben Sie 5 Tr. reines Neroliöl plus 7 Tr. der Bachblüte Crab Apple. Die Mischung verschütteln und vor der Arbeit dünn auf den Solarplexus auftragen.

Wasser-Ritual: Nach der Praxistätigkeit unter die Dusche gehen. Abseifen und Haare waschen sind nicht erforderlich, aber gründlich mit warmem Wasser duschen. Während das Wasser den Körper herabläuft, ein kleines Dankgebet dafür sprechen, dass der Luxus einer warmen Dusche zur Verfügung steht. Jetzt das Wasser bitten, alles, was nicht zu Ihnen gehört, mitzunehmen. Dann konzentrieren Sie sich auf die Erde und bitten diese, aus all den schweren Energien, die nun im Wasser sind, Kompost zu machen. Nach dem Duschen frische Kleidung anziehen - und den Feierabend genießen.

Analogiedenken altbewährter Therapierichtungen. Es verhilft zu einem Koordinatensystem, um die erhaltenen Informationen zu strukturieren. Strukturen helfen, den Überblick zu behalten und Informationslücken zu erkennen. Es ist der innere Blick auf diese Strukturen, der Assoziationen weckt und somit auch die Intuition fördert.

Folgen Sie den Analogien, die zu Ihrer angewandten Therapierichtung passen. Es spielt keine Rolle, ob Sie z. B. der TCM folgen oder europäischen Heilwegen. Wichtig ist nur, dass Sie ein in sich geschlossenes Analogie-System nutzen, nicht verschiedene Systeme mischen, und kritisch bleiben. Der Prüfstein ist und bleibt die faktische Realität.

Ich nutze das aus der Alchemie tradierte System der sieben planetaren Prinzipien. Damit kann ich die Einzigartigkeit jedes Patienten berücksichtigen, den zeitlichen Verlauf der Krankheit einschätzen, zuordnen, welche Therapierichtung für ihn im Augenblick geeignet ist, und zusätzlich auf der seelischen und geistigen Ebene unterstützende Maßnahmen finden.

\section{Dem Prozess vertrauen lernen}

Wir brauchen Mut und absolute Klarheit, um uns selbst auf den Prozess, durch den der Patient gehen wird, einzulassen. Wir brauchen Unerschrockenheit, um mit ihm hinabzusteigen in die Tiefen seiner Seele, seiner Empfindungen, seiner Emotionalität.

Wenn uns dieser Mut fehlt, ist unsere Sicht bei der fortlaufenden Diagnostik - der notwendigen Verlaufskontrolle jeder Therapie - getrübt. All jene Emotionen, die wir in uns selbst meiden oder verleugnen, können wir bei unserem Patienten nicht erkennen. Da Emotionen körperliche Entsprechungen haben und zudem physiologische Prozesse beeinflussen, werden diese nicht erkannt oder fehlinterpretiert.

Voraussetzung für diesen Mut ist jedoch die Klarheit, zu unterscheiden, was die eigene Emotion und was die Emotion des Patienten ist. Nur so gelingt es uns in jedem Augenblick der therapeutischen Arbeit, sei es in der Diagnostik oder Therapie, in Kontakt mit unserer Mitte zu bleiben. Von hier aus können wir die erforderlichen Entscheidungen treffen: Darf ich mich auf die
Emotionen meines Patienten einlassen, mich von ihnen berühren, aber nicht mitreißen lassen? Oder bin ich in dieser Situation besser beraten damit, bei Diagnostik und Therapie nur dem Verstand zu folgen.

Es geht hier nicht darum zu werten, was wertvoller ist: Inspiration oder Sachverstand. Es geht um die Klarheit des Therapeuten, in jedem Augenblick zu wissen, wo er selbst gefühlsmäßig steht und aus dieser Klarheit heraus den nächsten Schritt seiner Arbeit mit dem Patienten zu bestimmen.

\section{Eine Arbeit, die unter die Haut geht}

Sich einzulassen und das Leid des Patienten in sich selbst zu empfinden, wird von einigen psychologischen Schulen abgelehnt und von vielen Therapeuten gescheut. „Ich muss mich schützen“ wie oft habe ich diesen Satz von Kollegen gehört. Aus eigener Erfahrung weiß ich, dass es zu Beginn der Praxistätigkeit schwer ist, nach einem anstrengenden Tag alle Erlebnisse hinter sich zu lassen. C.G. Jung schreibt in seinem Buch „Psychologie und Alchymie“ zum Thema Übertragung/Gegenübertragung, dass diese unerlässlich ist, auch wenn sie den Therapeuten zeitweise belastet. Es ist eine Arbeit, die unter die Haut geht, denn keiner „rührt Gift an, ohne an gewissen undichten Stellen etwas davon abzubekommen; denn der wahre Arzt steht nirgends daneben, sondern überall darin." Dies gilt für Therapie wie Diagnostik gleichermaßen.

Nach einigen leidvollen Erfahrungen mit der Somatisierung von Patientensymptomen haben mir bestimmte Hilfsmittel (s. Kasten) auf dem Weg zu meiner Vollerwerbspraxis sehr geholfen. Diese führe ich seit 20 Jahren und ich kann mir keinen schöneren Beruf vorstellen. Es ist für mich eine Gnade, eine Aufgabe zu haben, die anderen Menschen hilft und mich anhält, meinen persönlichen Weg weiterzugehen. Und es ist gerade die Diagnostik, die mich immer wieder herausfordert, aktuelle Erkenntnisse und geistige Entwicklung miteinander zu verbinden.

\section{Dieser Artikel ist online zu finden: \\ http://dx.doi.org/10.1055/s-0035-1549135}

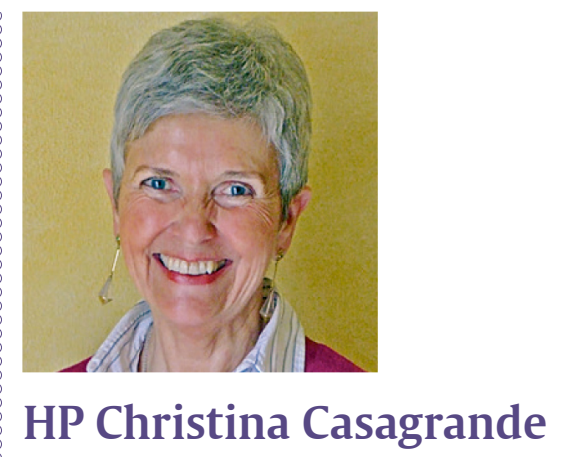

Mozartstr. 6 82299 Türkenfeld

E-Mail: email@christina-casagrande.de

Christina Casagrande ist Heilpraktikerin und arbeitet seit 1985 in eigener Praxis. Sie setzt neben den klassischen naturheilkundlichen Methoden hauptsächlich spagyrische Heilmittel nach Alexander von Bernus ein. Neben ihrer Praxisarbeit hält sie seit über 18 Jahren zum Thema Spagyrik Vorträge und Weiterbildungen für Kollegen und interessierte Laien. 